\title{
Principali riferimenti normativi sulle acque sotterranee in Piemonte.
}

\author{
Maria Governa \\ Regione Piemonte - Direzione Ambiente \\ Settore Tutela Quantitativa e Qualitativa delle Acque - Torino \\ maria.governa@regione.piemonte.it
}

\author{
Massimiliano Petricig \\ Regione Piemonte - Direzione Ambiente \\ Settore Ciclo Integrato dei Rifiuti e Servizio Idrico Integrato - Torino \\ massimiliano.petricig@regione.piemonte.it
}

Legge regionale 30/04/1996 n. 22 "Ricerca, uso e tutela delle acque sotterranee" e s.m.i. persegue la tutela preventiva del sistema idrico del sottosuolo e il corretto e razionale uso delle acque sotterranee anche attraverso l'acquisizione di conoscenze sistematiche delle medesime.

L'articolo 2, definite le nozioni di falda freatica e falde profonde, vieta la costruzione di opere che consentono la comunicazione tra i due sistemi sotterranei e stabilisce che la Giunta Regionale definisca i criteri tecnici per l'identificazione della base dell'acquifero superficiale, corredati da apposita cartografia, cui fare riferimento per l'applicazione delle disposizioni della predetta legge.

Per l'attuazione del sopraccitato adempimento con D.G.R. n. 34-11524 del 3/06/2009 - sono stati approvati i criteri tecnici per l'identificazione della base dell'acquifero superficiale e relativa cartografia, attualmente in fase di revisione.

Regolamento regionale 29/07/2003, n. 10/R recante: 'Disciplina dei procedimenti di concessione di derivazione di acqua pubblica (L.R. 61/2000)'.

Si applica a tutte le richieste di concessione di acque pubbliche superficiali e sotterranee, con esclusione, per quanto riguarda le acque sotterranee, dell'utilizzo domestico entro i limiti di portata massima di $2 \mathrm{~L} / \mathrm{s}$ e comunque per un prelievo massimo di $5000 \mathrm{mc} / \mathrm{a}$.

$\mathrm{E}^{\prime}$ in corso una revisione del regolamento volta a semplificare in generale l'iter di concessione, ricomprendendo, tra l'altro, nell'uso domestico l'alimentazione di impianti geotermici per il riscaldamento e il raffrescamento degli edifici e prevedendo una procedura semplificata per prelievo da pozzi con portate fino a $20 \mathrm{~L} / \mathrm{s}$ per il medesimo utilizzo geotermico e per la sostituzione dei pozzi non conformi o non utilizzabili per motivi tecnici.

Regolamento regionale 11/12/2006, n. 15/R recante: " $\mathrm{Di}$ sciplina delle aree di salvaguardia delle acque destinate al consumo umano (L.R. 61/2000)."

Con il quale si è inteso razionalizzare il procedimento amministrativo, graduare i vincoli e le limitazioni d'uso del suolo in maniera più efficace e coerente con le reali condizioni locali di vulnerabilità e rischio della risorsa idrica captata, nonché stabilire per tutte le captazioni nuove ed esistenti un legame diretto con la pianificazione di Settore riguardante il Servizio Idrico Integrato.

Disciplina inoltre le attività agricole ammissibili all'interno delle aree di salvaguardia in funzione delle condizioni idrogeologiche e pedologiche, prevedendo la predisposizione di uno specifico Piano di Utilizzazione Agricola delle aree circostanti le captazioni.

\section{Piano di Tutela delle Acque (PTA) - D.C.R. n. 117-10731 del 13/03/2007}

Strumento finalizzato al raggiungimento degli obiettivi di qualità dei corpi idrici e più in generale alla protezione dell'intero sistema idrico superficiale e sotterraneo piemontese. In attuazione della Direttiva 2000/60/CE "che istituisce un quadro per l'azione comunitaria in materia di acque" (Water Framework Directive), nonché della normativa nazionale di cui al decreto legislativo 152/1999, successivamente confluito nel decreto legislativo 152/2006, il PTA costituisce il documento di pianificazione generale contenente gli interventi volti a:

- prevenire e ridurre l'inquinamento e attuare il risanamento dei corpi idrici inquinati;

- migliorare lo stato delle acque ed individuare adeguate protezioni di quelle destinate a particolari usi;

- perseguire usi sostenibili e durevoli delle risorse idriche;

- mantenere la capacità naturale di autodepurazione dei corpi idrici, nonché la capacità di sostenere comunità animali e vegetali ampie e ben diversificate.

Il PTA è uno strumento dinamico che, sulla base delle risultanze del programma di verifica e dell'andamento dello stato di qualità, consente di aggiornare e adeguare di conseguenza l'insieme delle misure per il raggiungimento degli obiettivi in relazione a ciascuna area idrografica.

Una Relazione biennale della Giunta al Consiglio regionale trasmessa a novembre 2010 illustra i provvedimenti adottati, lo stato di attuazione delle misure di tutela e risanamento previste dal Piano e, conseguentemente, il programma di attività per le annualità successive, al fine di consentire all'Organo consiliare di formulare direttive e indirizzi per proseguire l'attività di attuazione del Piano. 
Bozza di Regolamento regionale recante: "Disciplina dell'installazione delle sonde geotermiche e del Registro regionale delle Sonde Geotermiche (L.R. 3/2009)".

E' finalizzato alla valorizzazione dell'utilizzo delle risorse geotermiche a bassa entalpia nel rispetto dell'ambiente e della tutela delle acque sotterranee e disciplina, in particolare, le modalità tecnico-operative per l'installazione e la gestione degli impianti e i contenuti minimi dei relativi progetti; i criteri tecnici e geologici per la localizzazione e l'installazione delle sonde geotermiche; le caratteristiche del Registro regionale delle Sonde Geotermiche (RSG) e le relative modalità di gestione. Non si applica agli impianti geotermici che comportano il prelievo e lo scarico di acqua, che restano disciplinati dalla vigente normativa statale e regionale in materia di derivazione, utilizzazione e scarico delle acque pubbliche sopra descrittto.

\section{Perché diventare socio A.N.I.P.A.?}

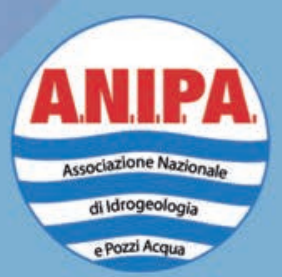

Offre un ottimo servizio di informazione e divulgazione, coordinamento delle attività di tutela delle imprese, supporto tecnico per le problematiche del cantiere, formazione del personale.

Compila la nostra scheda di adesione che troverai sul sito www.anipapozzi.it

Quota associativa annua:

- Imprese di perforazione pozzi $€ 150,001^{\circ}$ anno - $€ 300,00$ successivi

- Azienda produzione attrezzature, commerciale e servizi $€ 200,00$

- Professionista/Studio progettazione pozzi e indagini $€ 100,00$ 